\title{
Lyapunov exponent for the laser speckle potential: a weak disorder expansion
}

\author{
Evgeni Gurevich and Oded Kenneth \\ Department of Physics, Technion, Israel Institute of Technology, Haifa 32000, Israel
}

\begin{abstract}
Anderson localization of matter waves was recently observed with cold atoms in a weak $1 \mathrm{D}$ disorder realized with laser speckle potential [8]. The latter is special in that it does not have spatial frequency components above certain cutoff $q_{c}$. As a result, the Lyapunov exponent (LE), or inverse localization length, vanishes in Born approximation for particle wavevector $k>\frac{1}{2} q_{c}$, and higher orders become essential. These terms, up to the order four, are calculated analytically and compared with numerical simulations. For very weak disorder, LE exhibits a sharp drop at $k$ $=\frac{1}{2} q_{c}$. For moderate disorder (a) the drop is less dramatic than expected from the fourth order approximation and (b) LE becomes very sensitive to the sign of the disorder skewness (which can be controlled in cold atom experiments). Both observations are related to the strongly non-Gaussian character of the speckle intensity.

PACS numbers: 42.25Dd, 03.75.Nt, 72.15.Rn, 05.10.Gg
\end{abstract}

Technological progress in experiments with ultracold atoms provides an extraordinary level of control, allowing investigation of various quantum phenomena [1]. One such phenomenon is Anderson localization (AL) [2] of matter waves. Formulated originally to explain the absence of spreading of quantum-mechanical wave function in a disordered potential, it was recognized later as a common signature of wave propagation in random media, where waves may become exponentially localized because of the destructive interference between its multiple scattered components. Some indications of AL were observed with light [3], microwaves [4] and ultrasound [5], while in electronic systems it is hindered by finite temperature dephasing and interactions.

Cold atoms offer a unique possibility to study matter wave localization in conditions of very low temperature and tunable interactions (not available in solids), absence of absorption and controllable disorder. The latter may be introduced by different techniques, one of which is using static laser speckle, whereas potential felt by atoms is proportional to the speckle intensity with the sign of the detuning from the atomic transition [6]. Laser speckle, generated by passing expanded laser beam through diffusive plates, are special in that they have (i) exponential, i.e. strongly non-Gaussian, intensity distribution and (ii) finite support of their power spectrum [7]. As discussed below, these properties have strong effect on the localization properties in 1D systems.

Recently, AL was observed in Bose-Einstein condensates expanding in weak $1 \mathrm{D}$ disorder realized with laser speckle [8]. A simplified model for such experiments considers expansion of the condensate released from a harmonic trap as occurring in two stages [9], 10]: (1) explosive conversion of the interaction energy into kinetic energy, in which weak disorder may be neglected, followed by (2) expansion of the non-interacting gas in the disordered potential. The first stage ends up with a certain momentum distribution of the atomic cloud [11], which provides an initial condition for the second stage. Thus, the problem reduces to basically a single-particle localization, characterized in 1D by Lyapunov exponent (LE), or inverse localization length [12]. The latter, in a weak 1D speckle disorder with a spatial frequency cutoff $q_{c}$, has some peculiarities, because in 1D (as opposed to higher dimensions), elastic scattering is a "binary" process: either the particle wavevector $k$ remains unchanged (forward scattering), or it reverses its sign and changes by the amount of $2 k$ (backscattering). As a result, backscattering amplitude (in a single potential realization) vanishes in the Born approximation for $2 k>q_{c}$, while in higher approximations, of order $n$, it vanishes for $2 k>n q_{c}$. Correspondingly, in weak speckle disorder, LE is expected to exhibit a series of cascading drops at $k=\frac{n}{2} q_{c}$, so called "effective mobility edges". Thus, in order to assess the localization properties for $2 k>q_{c}$, one should know LE beyond the Born approximation, which is a subject of this paper.

LE in 1D disorder was studied extensively for a variety of continuous and discrete models, which usually dealt with an uncorrelated disorder or a specific type of correlation (see Ref. 12] and reference herein). For arbitrary correlation and in the weak disorder Born approximation, $\mathrm{LE}$ is known to be proportional to the disorder power spectrum [12], [15]. In the recent years, correlations in 1D disorder attracted much attention because they can induce unusual localization properties, such as existence of extended states [13], deviation from the single parameter scaling (SPS) [14] and appearance of the "effective mobility edge" when LE vanishes in Born approximation [15],[16]. The latter means that exact localization length would exceed system size for sufficiently weak disorder, while higher orders are required to specify this regime quantitatively [16].

In this paper we report a systematic weak disorder expansion for LE for two orders beyond Born approximation, which is then applied to the laser speckle disorder [17. The analytical study is verified by numerical simulations, which also allow examination of regimes beyond weak disorder. The author of Ref.[16] performed a weak disorder expansion for a "generalized" LE. Let us stress that, although the generalized LE is easier to compute, it is the standard LE, studied here, that is of prime interest 
in the localization problem. While these two quantities coincide in the lowest order [16], they differ in higher orders (see below). More generally, the equality holds under the assumption of SPS, and the differences found show how the latter is affected by disorder correlations.

We consider continuous one-dimensional model

$$
\frac{d^{2} \psi}{d x^{2}}+k^{2}(1+\eta(x)) \psi=0
$$

where $\eta(x)$ is the dimensionless disordered potential (for a quantum particle, $\eta(x)=-2 m V(x) / \hbar^{2} k^{2}$ is the ratio of the potential $V(x)$ to the particle energy). We assume zero mean finite-range correlated disorder, specified by its (joint) cumulants $(n=2,3, \ldots)$

$$
\kappa_{n}\left(x_{1}, \ldots, x_{n-1}\right)=\left(\frac{g}{2 R_{c}}\right)^{\frac{n}{2}} \Gamma_{n}\left(\frac{x_{1}}{R_{c}}, \ldots, \frac{x_{n-1}}{R_{c}}\right),
$$

where $R_{c}$ is the correlation scale, the dimensionless functions $\Gamma_{n}\left(x_{1}, \ldots, x_{n-1}\right)$ decay on the scale of unity and $g$ is noise intensity. One chooses $\int_{0}^{+\infty} \Gamma_{2}(x) d x=1$, so that the limit $R_{c} \rightarrow 0$ yields Gaussian white noise with the two-point correlation $\left\langle\eta(x) \eta\left(x^{\prime}\right)\right\rangle=g \delta\left(x-x^{\prime}\right)$.

LE is defined as

$$
\lambda=\lim _{x \rightarrow \infty}(2 x)^{-1}\left\langle\ln \left(k^{2}|\psi(x)|^{2}+\left|\psi^{\prime}(x)\right|^{2}\right)\right\rangle,
$$

where $\langle.$.$\rangle denotes the disorder average, while the$ generalized LE considered in Ref.[16] is given by $\lim _{x \rightarrow \infty} \frac{1}{4 x} \ln \left\langle k^{2}|\psi(x)|^{2}+\left|\psi^{\prime}(x)\right|^{2}\right\rangle$. We calculate the LE (3) using the phase formalism relation [12]

$$
\lambda=\int z P_{s t}(z) d z
$$

where $z=\psi^{\prime} / \psi$ and $P_{s t}(z)$ is the stationary (i.e. the $x \rightarrow$ $\infty$ limit) distribution of $z$. Introducing phase $\theta$, defined by $z=-k \tan (\theta / 2)$ and obeying "evolution" equation

$$
\frac{\partial \theta}{\partial x}=2 k+2 k \cos ^{2}(\theta / 2) \eta(x),
$$

LE is expressed in terms of the stationary distribution of the phase as

$$
\lambda=-k \int_{-\pi}^{\pi} \tan \left(\frac{\theta}{2}\right) P_{s t}(\theta) d \theta .
$$

Then, calculating the weak disorder expansion for $P_{s t}(\theta)$ and substituting it into (6), yields the required expansion for the LE $\lambda$. In the case in which $\eta(x)$ in (5D) is a $\delta$-correlated process, one can use standard technique [18] to write down a Fokker-Plank equation for $P(\theta ; x)$. When $\eta(x)$ is a correlated process, the method of "ordered cumulants" [19] can be used to obtain an approximate master equation for $P(\theta ; x)$, given by a perturbative expansion in powers of $\beta \gamma^{1 / 2}$, where the dimensionless parameters

$$
\gamma=2 k R_{c} \text { and } \beta=\sqrt{g k / 8},
$$

describe disorder correlation scale and strength respectively. Specific application of the method to the present problem involves many technical details, which will be given elsewhere [20]. Here we only outline the main steps of the derivation. Assuming small $\beta$, but arbitrary $\gamma$, the following formal expansion of the master equation for $P(\theta ; x)$ is obtained

$$
\frac{\partial}{\partial x} P(\theta ; x)=\left[A_{0}+2 \sum_{n=2}^{\infty} \beta^{n} K_{n}\right] P(\theta ; x) .
$$

$K_{n}$ are differential operators, whose definition involves integration over various ordered products of the operator $\tilde{A}_{1}(x) \equiv e^{x A_{0}} A_{1} e^{-x A_{0}}=-2 k \partial_{\theta} \cos ^{2}(\theta-k x)$, weighted with combinations of the cumulant functions $\Gamma_{m \leq n}$ (2). Here operators $A_{0}=-2 k \partial_{\theta}$ and $A_{1}=-2 k \partial_{\theta} \cos ^{2}(\theta / 2)$ are related respectively to the deterministic and the stochastic terms on the right side of (5).

Next, in the stationary limit, the master equation for $P(\theta ; x)$ reduces to an ordinary differential equation for $P_{s t}(\theta)=\lim _{x \rightarrow \infty} P(\theta ; x)$ and one looks for a perturbative solution

$$
P_{s t}(\theta)=\sum \beta^{n} P_{n}(\theta) .
$$

Let us note that this "double-stage" perturbation approach is inconsistent in some cases [21],22], such as when $P_{s t}(\theta)$ is singular in the limit $\beta \rightarrow 0$. This, however, does not occur in our case, since $P_{s t}(\theta)$ becomes uniform for $\beta \rightarrow 0$. Thus, substitution of the solution (9) into (6) yields the required LE expansion

$$
\lambda=k \sum_{n \geq 2} \beta^{n} \lambda_{n}(\gamma) .
$$

The first four coefficients are given by

$$
\lambda_{2}=c_{o}, \lambda_{3}=c_{6}, \lambda_{4}=c_{0}\left(c_{1}-c_{2}\right)+c_{3} c_{1}+2 c_{4}+c_{5},
$$

where $c_{i}$ are the following functions of $\gamma\left(=2 k R_{c}\right)$ :

$$
\begin{aligned}
c_{0}= & \int_{0}^{\infty} d s \Gamma_{2}(s) \cos \gamma s=\frac{\tilde{\Gamma}_{2}(\gamma)}{2}, \quad c_{3}=-\gamma \frac{\partial c_{0}}{\partial \gamma}, \quad(12) \\
c_{1}= & \int_{0}^{\infty} d s \Gamma_{2}(s) \sin \gamma s=\int_{-\infty}^{+\infty} \frac{d q}{2 \pi} \frac{\tilde{\Gamma}_{2}(q)}{\gamma-q}, c_{2}=\gamma \frac{\partial c_{1}}{\partial \gamma}, \\
c_{4}= & \int_{0}^{\infty} d s_{1} \int_{0}^{s_{1}} d s_{2} \Gamma_{2}\left(s_{1}\right) \Gamma_{2}\left(s_{2}\right) \times \\
& \times\left[2 \sin \left(\gamma s_{1}\right)-\gamma\left(s_{1}-s_{2}\right) \cos \left(\gamma s_{1}\right)\right] \\
= & \int \frac{d q}{2 \pi} \tilde{\Gamma}_{2}(q)\left[\frac{\tilde{\Gamma}_{2}(\gamma-q)}{q}+\gamma \frac{\tilde{\Gamma}_{2}(\gamma-q)-\tilde{\Gamma}_{2}(\gamma)}{2 q^{2}}\right], \\
c_{5}= & -\gamma \int_{0}^{\infty} d s_{1} \int_{s_{1}}^{\infty} d s_{2} \int_{s_{2}}^{\infty} d s_{3} \Gamma_{4}\left(s_{1}, s_{2}, s_{3}\right) \times \\
& \times\left(2 \cos \left(\gamma s_{3}\right)+\cos \left(\gamma s_{1}-\gamma s_{2}-\gamma s_{3}\right)\right),
\end{aligned}
$$




$$
\begin{aligned}
c_{6} & =-\sqrt{2 \gamma} \int_{0}^{\infty} d s_{1} \int_{s_{1}}^{\infty} d s_{2} \Gamma_{3}\left(s_{1}, s_{2}\right) \sin \gamma s_{2} \\
& =\frac{\sqrt{2 \gamma}}{4 \pi} \int \frac{d q}{q}\left[\tilde{\Gamma}_{3}(q, \gamma)-\tilde{\Gamma}_{3}(q, \gamma-q)\right],
\end{aligned}
$$

and $\tilde{\Gamma}_{n}\left(q_{1}, \ldots, q_{n-1}\right)$ is a Fourier transform of $\Gamma_{n}\left(x_{1}, \ldots, x_{n-1}\right)$. The coefficients $c_{i}$ for $i=5,6$ may be tagged "non-Gaussian", since they depend on the higher (non-Gaussian) cumulants $\Gamma_{3}$ and $\Gamma_{4}$ only, and vanish for Gaussian disorder. Note that $c_{i=0,3,4,6}$ would vanish for $\gamma$ above certain threshold, if $\tilde{\Gamma}_{2}(q)$ and $\tilde{\Gamma}_{3}\left(q_{1}, q_{2}\right)$ have finite support (same applies to $c_{5}$, whose expression in terms of $\tilde{\Gamma}_{4}$ is not shown). Besides, if $\tilde{\Gamma}_{2}(q)$ or its derivative are discontinuous at some point $q_{c}$, then $c_{4}(\gamma)$ would diverge at $\gamma=q_{c}$ and the perturbation theory would break down for this $\gamma$ (see below).

As expected, $\lambda_{2}$, the lowest order coefficient in (10), coincides with earlier results [12]. Comparing our results for the standard LE to those of Ref.[16] for the generalized LE (even orders only), one finds that the two coincide in the second and vary in the fourth order, though the difference exists only for non-Gaussian disorder. Namely, the corresponding non-Gaussian terms $c_{5}(\gamma)$ in $\lambda_{4}$ have different expressions, which at small $\gamma$ scale differently with $\gamma$ and have opposite signs. Thus, the SPS relations (see e.g. Ref.[14]) hold up to the order four in Gaussian, while are broken in non-Gaussian disorder.

So far our results are quite general and pertain to an arbitrary random potential, the only condition being sufficiently fast decay of the disorder cumulants [19]. Now we specialize to the case of a laser speckle potentials, produced by transmitting laser beam through a diffuser with a rectangular aperture [7], 6]. Its intensity pair correlation function is $\Gamma_{2}(x)=\frac{2}{\pi} \frac{\sin ^{2} x}{x^{2}}$, whose Fourier transform $\tilde{\Gamma}_{2}(q)$ vanishes for $|q| \geq 2\left(\tilde{\Gamma}_{2}(q)\right.$ is related to the shape of the optical aperture, therefore for arbitrary but finite aperture it would always have finite support [7]). Assuming that speckle field (as opposed to intensity) is a complex Gaussian variable, which is true for sufficiently large diffuser [7], one concludes that any $\Gamma_{n}$ is expressed solely in terms of "irreducible" products of the two-point field correlators $\tilde{w}\left(x_{i}-x_{j}\right)$. For example, the $3^{\text {rd }}$ cumulant is $\Gamma_{3}\left(x_{1}, x_{2}\right)=-2 \varepsilon\left(\frac{2}{\pi}\right)^{3 / 2} \tilde{w}\left(x_{1}\right) \tilde{w}\left(x_{1}-x_{2}\right) \tilde{w}\left(x_{2}\right)$, where $\tilde{w}(x)=\frac{\sin x}{x}$ is related to the Fourier transform of the "rectangle" function and $\varepsilon= \pm 1$ is the sign of the disorder distribution skewness, depending on either "blue" or "red" laser detuning from the atomic transition.

Substituting the explicit expressions for $\Gamma_{n}$ into the definitions of the coefficients $c_{i}$, one obtains the following LE expansion coefficients for the speckle disorder:

$$
\begin{aligned}
& \lambda_{2}=\frac{2-\gamma}{2} \chi(2-\gamma), \quad \lambda_{4}=\left(\lambda_{4}^{G}+\lambda_{4}^{N G}\right) \chi(4-\gamma), \\
& \lambda_{3}=2 \varepsilon \sqrt{\frac{\gamma}{\pi}}\left[(\gamma-2) \ln \frac{2-\gamma}{2}-\gamma \ln \frac{\gamma}{2}\right] \chi(2-\gamma),
\end{aligned}
$$

where $\lambda_{4}^{G} \equiv c_{0}\left(c_{1}-c_{2}\right)+c_{3} c_{1}+2 c_{4}$ and $\lambda_{4}^{N G} \equiv c_{5}$ denote the "Gaussian" and the non-Gaussian parts of $\lambda_{4}$ and $\chi(x)$ is the Heaviside step function. For $\gamma \leq 2$

$$
\begin{aligned}
\lambda_{4}^{G}= & \frac{1}{2 \pi}\left[4 \gamma-3 \gamma^{2}+\left(\frac{\gamma^{2}}{2}+2\right) \ln \frac{\gamma+2}{2}+\right. \\
& \left.+\left(\frac{3 \gamma^{2}}{2}-10\right) \ln \frac{2-\gamma}{2}+\gamma(\gamma-4) \ln \frac{\gamma}{2}\right], \quad(17) \\
\lambda_{4}^{N G} \approx & \frac{\gamma \pi}{3}+\frac{\gamma}{\pi}(2-\gamma) \ln (2-\gamma)\left(\frac{11}{2} \ln \frac{2-\gamma}{4 e^{2}}-\ln 2\right)
\end{aligned}
$$

while for $2<\gamma<4$

$$
\begin{aligned}
\lambda_{4}^{G} & =\frac{1}{2 \pi}\left[\gamma^{2}-4 \gamma-\left(\gamma^{2}-4 \gamma+8\right) \ln \frac{\gamma-2}{2}\right], \\
\lambda_{4}^{N G} & =\frac{\gamma^{2}}{\pi}\left[2 \operatorname{Li}_{2} \frac{2}{\gamma}-\frac{\pi^{2}}{6}+\ln ^{2} \frac{\gamma}{2}\right]-\frac{\gamma(\gamma-2)}{\pi} \ln ^{2} \frac{\gamma-2}{2},
\end{aligned}
$$

where $\operatorname{Li}_{2}(z)$ is the dilogarithm and $\lambda_{4}^{N G}$ for $\gamma<2$ was calculated approximately, assuming $(2-\gamma) \ll 1$ and neglecting $\mathcal{O}(2-\gamma)$ terms (exact calculation is too lengthy to carry out, while $\lambda_{4}^{N G}$ is important only close to or above $\gamma=2)$. The coefficients $\lambda_{i}(\gamma)$ are plotted in the insert of Fig. 2, As already noticed [6], $\lambda_{2}$ vanishes for $\gamma=2 k R_{c}>2$. Then, as can be expected from the general perturbation theory for scattering, $\lambda_{3}$ and $\lambda_{4}$ vanish for $\gamma>2$ and $\gamma>4$ respectively. Thus, for $2<\gamma<4$, LE switches from quadratic to quartic leading dependence on $\beta$ and, for weak disorder, undergoes a steep decrease at $\gamma=2 k R_{c}=2$. Note that for speckle potential our perturbation theory breaks down at $\gamma=2$, where $\lambda_{4}^{G}$ has a logarithmic divergence. As explained above, this is because $\tilde{\Gamma}_{2}(q)=(2-|q|) \chi(2-|q|)$ has a discontinuous derivative at $q=2$.

Our analytical study was supplemented with numerical simulations of the discrete tight-binding model near the energy band edge, where it is a good approximation to continuous problem. LE was computed using the transmission matrix formalism [23]. LE, as a function of $\gamma$ at fixed $\eta_{0} \equiv \sqrt{\left\langle\eta^{2}\right\rangle}$, the ratio between the disorder standard deviation and the particle energy, is shown in Fig. 1 for $\eta_{0}=0.08$ and $\eta_{0}=0.15$ (the disorder strength parameter $\beta$, rewritten as $\beta=\frac{1}{4} \sqrt{\pi \gamma} \eta_{0}$, changes along with $\gamma$ in this parametrization). LE, for each $\eta_{0}$ computed for both signs of $\varepsilon$, is significantly larger for $\varepsilon=+1$, which demonstrates strong effect of the non-Gaussian character of the speckle disorder. While agreement between the analytical and the numerical results is acceptable for $\gamma<2$, it appears to be very poor for $\gamma>2$, which is because the disorder is not weak enough. To clarify this point, numerical LE $\left(\lambda^{(\mathrm{Num})}\right)$, computed for different values of $\beta$ and $\varepsilon$ at fixed $\gamma=2.2$ and divided by the analytical $\lambda=k \beta^{4} \lambda_{4}$, was fitted to a $4^{t h}$ degree polynomial of $\varepsilon \beta$, as appears in Fig. 2 together with the fit equation (recall that for $\gamma>2$ the perturbative expansion (10) for $\lambda$ starts with the fourth order term). The free constant of the fit is $\lambda_{4}^{(\mathrm{Num})} / \lambda_{4}$, and its value 1.003 indicates excellent agreement between the perturbation theory and numerics. On the other hand, the fit equation in Fig. 


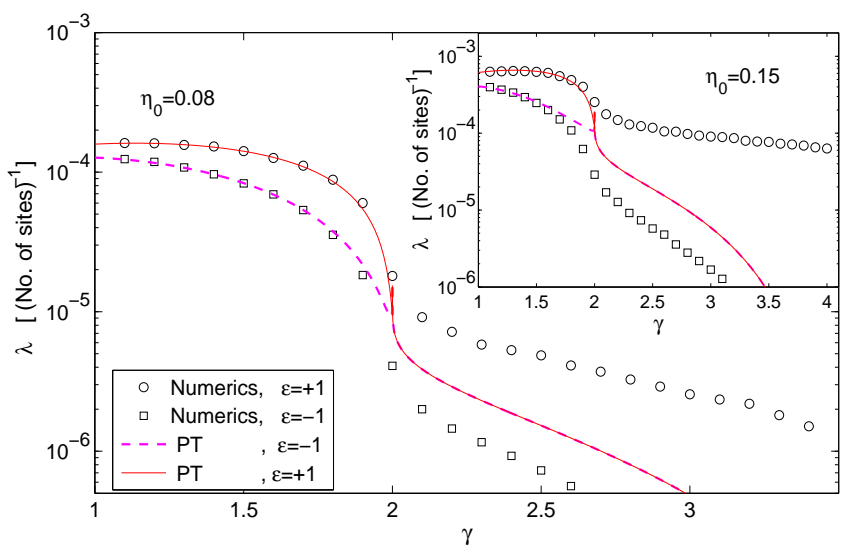

FIG. 1: (color online) Lyapunov exponent $\lambda$ for $\eta_{0}=0.08$ (main panel) and $\eta_{0}=0.15$ (insert) as a function of the dimensionless disorder correlation parameter $\gamma=2 k R_{c}$. Circles and squares show numerical data for positive $(\varepsilon=+1)$ and negative $(\varepsilon=-1)$ skewness of disorder, while solid and dashed lines give corresponding $4^{\text {th }}$ order expansion. Curve's spike at $\gamma=2$ reflects the logarithmic divergence of $\lambda_{4}^{G}$.

2 shows that the $4^{\text {th }}$ order approximation is acceptable only for very weak disorder $(\beta \ll 0.1)$. Repeating similar test for $\gamma \approx 2$, we conclude that the logarithmic peak of $\lambda_{4}^{G}$ (Fig. 2, insert) is reproduced in the numerics up to the smearing effect of the finite system size $L$ (this can be accounted for by smoothing $\lambda_{i}(\gamma)$ over $\left.\Delta \gamma \sim \frac{R_{c}}{L}\right)$.

Note the very rapid growth of the higher order coefficients of the fit in Fig. 2, corresponding to higher orders in LE expansion. As suggested by the structure of the perturbation theory, it is related to the non-Gaussian character of the exponential distribution of the speckle intensity, whose cumulants grow factorially. This explains both the failure of the $4^{t h}$ order approximation and the strong dependence of LE on the sign of the disorder skewness $\varepsilon$ found at moderately weak disorder (Fig. 11). The perturbative expansion up to order $\beta^{4}$ accounts for the effect of $\varepsilon$ only for $\gamma<2$, since its only odd term $\lambda_{3}$ vanishes for $\gamma>2$. Therefore, analytical curves for $\varepsilon=+1$ and $\varepsilon=-1$ coincide for $\gamma>2$ (Fig. 1).

Finally, we address persistence of the effective mobility edge at $\gamma=2$ as a function of the disorder strength. This question is important for the recent experiments on the BEC expansion [8] (with $\varepsilon=+1$ ), because, if distribution of the atomic $k$ 's in the "exploded" condensate stretches beyond the speckle frequency cutoff $2 / R_{c}$, then the deepness of the LE drop at $\gamma=2$ becomes crucial for predicting algebraic versus exponential decay of density profiles [9]. Our computations in Fig. 1 show that this drop depends strongly on the disorder strength and becomes of effectively one order of magnitude or less for $\eta_{0} \gtrsim 0.1$ (relevant to the experimental regimes [8]). In addition, note the difference in LE for "blue" $(\varepsilon=+1)$ and "red" $(\varepsilon=-1)$ detuning: for negative detuning LE is smaller, but its relative variation across $\gamma=2$ is larger.

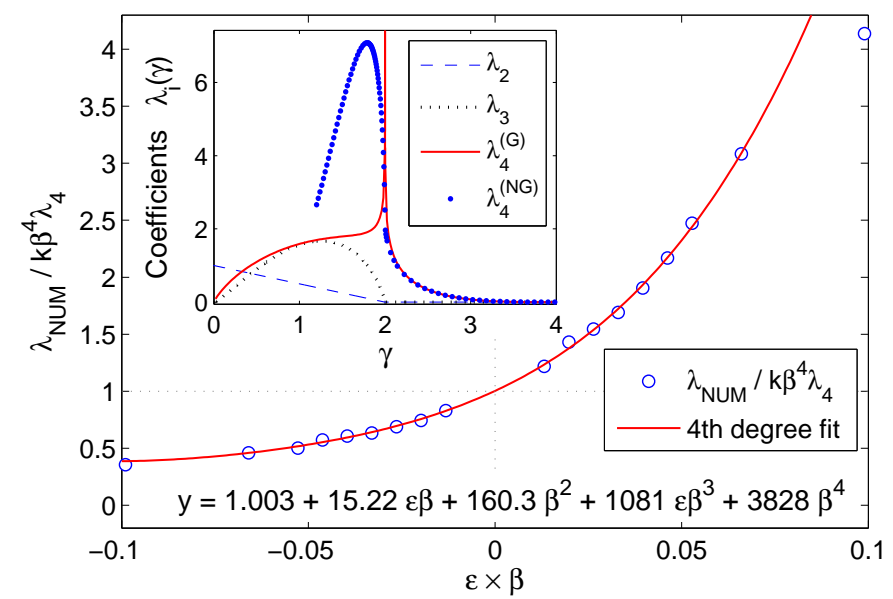

FIG. 2: (color online) LE calculated at fixed $\gamma=2.2$ as a function of disorder strength and "sign" $\varepsilon \beta$. Circles represent numerical LE devided by $k \beta^{4} \lambda_{4}$. Line and equation show the $4^{t h}$ degree fit over all the points except the left- and the right-most ones. Insert: expansion coefficients $\lambda_{i}(\gamma)$.

(Though our parametrization is not natural for this experiment - we fixed $k$ with disorder amplitude and varied $R_{c}$, instead of varying $k$ with the rest fixed, - the conclusions remain valid, since mainly the behavior near $\gamma=2$ is concerned.)

In conclusion, we derived general expansion for the Lyapunov exponent (LE) in 1D correlated disorder two orders beyond the Born approximation. Comparing it with that for the generalized LE [16] shows that single parameter scaling is broken perturbatively in the fourth order for non-Gaussian disorders (with finite moments). Applying this expansion to speckle disorder with Fourier spectrum cutoff $q_{c}=\frac{2}{R_{c}}$, we find that the leading order dependence of LE on the disorder strength crosses from quadratic for $k R_{c}<1$ to quartic for $1<k R_{c}<2$, as expected from the standard QM perturbation theory. For very weak disorder, this results in large and steep drop of LE across $k R_{c}=1$. For larger but still weak disorder, this drop moderates, while LE becomes very sensitive to the skewness of the disorder distribution (for $k R_{c} \gtrsim 1$ ). This is because of strongly non-Gaussian distribution of speckle intensity, whose cumulants grow factorially fast. Physically, this reflects non-perturbative contribution of rare but large potential peaks (dips) of the typical width $R_{c}$ to the scattering process.

\section{Acknowledgments}

E.G. thanks B. Shapiro for suggesting the problem and for his guidance. We wish to thank K. Mallick for valuable discussions and are grateful to A. Aspect for an illuminating discussion and for informing us about the work on correlated random potentials carried on in his and col- 
laborating groups [17]. This research was supported by
ISF grant.
[1] For a recent review, see I. Bloch et al., Rev. Mod. Phys 80, 885 (2008)

[2] P. W. Anderson, Phys. Rev. 109, 1492 (1958).

[3] D. S. Wiersma et al., Nature 390, 671673 (1997); T. Schwartz et al., Nature 446, 5255 (2007); Y. Lahini et al., Phys. Rev. Lett. 100, 013906 (2008).

[4] Chabanov et al., Nature 404, 850853 (2000).

[5] H. Hu et al., Nature Physics 4, 945 (2008).

[6] D. Clément et al., New J. Phys 8, 165 (2006); L. SanchezPalencia et al., New J. Phys 10, 045019 (2008).

[7] J.W. Goodman, "Statistical properties of Laser Speckle Patterns" in "Laser Speckle and Related Phenomena", edited by J.C. Dainty, 2nd Ed., Springer-Verlag 1984.

[8] J. Billy et al., Nature 453, 891 (2008).

[9] L. Sanchez-Palencia et al., Phys. Rev. Lett. 98, 210401 (2007).

[10] B. Shapiro, Phys. Rev. Lett. 99, 060602 (2007).

[11] Yu. Kagan et al., Phys. Rev. A 54, R1753 (1996); Y. Castin and R. Dum, Phys. Rev. Lett. 77, 5315 (1996).

[12] I.M. Lifshitz et al., Introduction to the Theory of Disordered Systems (Wiley, New York, 1988).

[13] D. H. Dunlap et al., Phys. Rev. Lett. 65, 88 (1990);
F.A.B.F. de Moura and M. L. Lyra, Phys. Rev. Lett. 81, 3735 (1998); P. Carpena et al., Nature 418, 955 (2002); A. M. Garcia-Garcia and E. Cuevas, arXiv:0808.3757 1.

[14] M. Titov and H. Schomerus, Phys. Rev. Lett. 95, 126602 (2005).

[15] F.M. Izrailev and A.A. Krokhin, Phys. Rev. Lett. 82, 4062 (1999).

[16] L. Tessieri, J.Phys. A: Math. Gen. 35, 9585-9600 (2002).

[17] When this paper was in final preparation, we were informed about an independent study of the LE in the laser speckle potential, A. Aspect, private communication and P. Lugan et al., arXiv:0902.0107v2.

[18] H. Risken, The Fokker-Planck Equation, second edition (Springer-Verlag, Berlin, 1989).

[19] N. G. van Kampen, Stochastic Processes in Physics and Chemistry, revised and enlarged edition (North-Holland, Amsterdam, 1992).

[20] paper in preparation

[21] P. Hanggi and P. Jung, Adv. Chem. Phys. 89, 239 (1995).

[22] N. G. van Kampen, J. Stat. Phys. 54, 1289 (1989).

[23] Y. Liu and K.A. Chao, Phys. Rev. B 34, 5247 (1986). 